\title{
Depressão no trabalho da enfermagem: revisão sistemática de literatura*
}

\section{Depression in nursing work: a systematic review}

Luana Aparecida Lima Ferreira' Lucas Lima Ferreira ${ }^{2}$
* Recebido em: 06/05/2014. Aprovado em: 25/05/2015.

1 Acadêmica do curso de Psicologia da Fundação Educacional de Fernandópolis - FEF.

2 Mestrado em Fisioterapia pela Universidade Estadual Paulista - UNESP. Docente do curso de Fisioterapia - UNILAGO. Fisioterapeuta Intensivista do Hospital de Base - HB. E-mail: lucas_lim21@hotmail.com.

\section{Resumo}

A profissão de enfermagem está predisposta a vários riscos físicos e psicológicos, entre estes, a depressão, que, pode acarretar o absenteísmo no trabalho. O objetivo desta pesquisa foi identificar estudos que analisaram a ocorrência de depressão relacionada ao trabalho de enfermagem, a fim de nortear estratégias de prevenção e enfrentamento junto a esses profissionais. Tratou-se de revisão sistemática de literatura por meio do levantamento de pesquisas publicadas entre os anos de 2000 a 2010, com disponibilidade de texto completo livre, nas bases de dados eletrônicas Lilacs, SciELO e MedLine. Foram identificados sete estudos, que apresentaram grau de depressão ou comprometimento da saúde mental dos profissionais de enfermagem e que esta pode ser influenciada por fatores internos e externos ao trabalho. Concluiu-se que a depressão, como problema de saúde, tem acometido, com frequência, os trabalhadores de enfermagem, porém houve escassez de estudos relacionados ao tema, publicados na última década.

Palavras-chave: Depressão. Enfermagem. Trabalho. Saúde ocupacional.

\section{Abstract}

The nursing profession is predisposed to various physical and psychological risks, among these, the depression, which can lead to absenteeism at work. The objective of this research was to identify studies that examined the occurrence of depression related to nursing work in order to guide prevention strategies and coping among these professionals. It was a literature systematic review through survey research published between the years 2000 to 2010, with free full-text availability, in electronic databases Lilacs, SciELO and MedLine. We identified seven studies, which showed some degree of impairment of depression or mental health nurses and this can be influenced by internal and external work. Was concluded that depression as a health problem has affected often-nursing workers; however, there was a lack of studies related to the topic, published in the last decade. Keywords: Depression. Nursing. Work. Occupational health. 


\section{Introdução}

A área da saúde ocupacional mental (SOM) tem apresentado vertiginoso interesse e crescimento no campo das pesquisas científicas nas últimas décadas, levando inúmeros periódicos nacionais e internacionais a publicar cada vez mais pesquisas relacionadas ao tema, com abordagens em diversas corporações, como hospitais, escolas, fábricas e universidades (BABA; GALAPERIN; LITUCHY, 1999). Esse interesse se deu pelo expressivo aumento da prevalência de transtornos psicológicos, tanto na população em geral quanto na parcela economicamente ativa desta, pois, esse incremento, gerou, entre outros, altos custos sociais (BOUBONNAIS et al., 1998). Os profissionais mais susceptíveis aos problemas de SOM são aqueles que interagem, a maior parte do tempo, com pessoas que necessitam de sua ajuda, como os enfermeiros, os professores, as assistentes sociais, entre outras profissões (BABA; GALAPERIN; LITUCHY, 1999).

Diversos fatores estressores específicos do trabalho, já foram identificados, entre eles, o clima de trabalho negativo, falta de clareza nas tarefas executadas e de expectativas de crescimento profissional e ascensão social, refletindo em efeitos adversos sobre a saúde dos profissionais, principalmente os que trabalham no âmbito da saúde (SCHAEFER; MOOS, 1996). As pressões no trabalho, como o conflito de interesses e a sobrecarga, contribuem para o desequilíbrio e estresse, que levam à deterioração da saúde mental manifestada principalmente pela depressão (BOUBONNAIS et al., 1998). A literatura reporta vários estudos relacionados à depressão, porém, a prevalência desse problema entre os profissionais de enfermagem, tem sido pouco estudada (FRANCO; BARROS; MARTINS, 2005).

Algumas pesquisas abordaram aspectos como a ansiedade, o estresse e a síndrome de Burnout do enfermeiro, em suas diversas áreas de atuação (JODAS; HADDAD, 2009; PAFARO; MARTINO, 2004; BORGES; CARLOTTO, 2004), apontando para indícios de alterações na saúde emocional desses trabalhadores, sem, contudo, identificar os fatores desencadeantes desses distúrbios. Entretanto, observa-se uma clara subestimação do problema da depressão sobre os profissionais da enfermagem no que tange à literatura científica, suscitando a necessidade de maiores investigações desse tema, principalmente, mediante a constatação empírica do aumento do número de trabalhadores de enfermagem com depressão (FRANCO; BARROS; MARTINS, 2005).

O aumento do número de trabalhadores da saúde com transtornos mentais tem levado à implementação de programas de atenção à saúde do trabalhador, que, entre outras providências, tem-se observado a ampliação da atuação de serviços como o de psicologia e psiquiatria em ambientes hospitalares, que, além de prestarem atendimento às pessoas internadas e em atendimento ambulatorial, assistem os empregados dessas instituições (ELIAS; NAVARRO, 2006).

Diante disso, o presente estudo teve por objetivo identificar, por meio de uma revisão sistemática da literatura científica, estudos que analisaram a ocorrência de depressão relacionada ao trabalho de enfermagem, a fim de mapear estratégias de prevenção e enfrentamento junto a esses profissionais.

\section{Metodologia}

\subsection{Seleção e características dos estudos}

Tratou-se de revisão sistemática de literatura com a aplicação de estratégia de busca a artigos científicos selecionados com base em consulta às bases de dados Literatura Latino Americana e do Caribe em Ciências da Saúde (Lilacs), Scientific Electronic Library Online (SciELO) e National Library of Medicine (Medline/via PubMed), com artigos publicados no período entre 2000 a 2010. Para a busca, foram utilizadas as palavras-chave: depressão, enfermagem, saúde mental, saúde ocupacional e trabalho, os quais foram definidos com base nos Descritores em Ciências da Saúde (DeCS), bem como seus correspondentes na língua inglesa: depression, nursing, mental health, occupational health e work, tomando-se por base o Medical Subjetic Headings (MeSH).

Outra estratégia utilizada foi a busca manual nas listas de referências dos estudos selecionados para complementação da pesquisa. Todas as etapas da busca foram realizadas por somente um pesquisador, sob a supervisão de outro pesquisador mais experiente.

\subsection{Critérios de inclusão e exclusão}

Foram inclusos artigos científicos nacionais e internacionais (inglês e espanhol), publicados entre o período de janeiro de 2000 a dezembro de 2010, refe- 
rentes à depressão relacionada ao trabalho de enfermagem, com disponibilidade de texto completo livre.

Foram excluídas dissertações ou teses acadêmicas, artigos de revisão, publicações em idiomas diferentes aos dos inclusos, publicações anteriores a $2000 \mathrm{e}$ posteriores a 2010, com estudo da população ou amostra não correspondente aos profissionais de enfermagem e/ou não disponíveis no Brasil, na íntegra ou por meio eletrônico.

\subsection{Estratégia de seleção}

Para a seleção dos artigos, inicialmente foi realizada a triagem dos títulos relacionados ao tema em questão. Essa seleção foi baseada nos títulos que abordassem como ideia principal: a depressão no trabalho da enfermagem. Ao final da busca, foram excluídos os títulos repetidos, já que esta foi realizada em diversas bases de dados. Em seguida, foi feita a leitura detalhada dos resumos dos artigos, a fim de selecionar aqueles que abordassem exclusivamente métodos de avaliação da depressão na atividade laboral de profissionais de enfermagem. Excluídos os resumos que não versavam sobre o tema, os textos completos foram avaliados e os que não se enquadravam nos critérios de exclusão foram inclusos como resultado final da busca.

\subsection{Análise dos dados}

Foi realizada a análise de conteúdo dos estudos encontrados e os dados foram agrupados de forma qualitativa e apresentados na forma de tabela com a descrição das seguintes características: autor e ano do estudo, população estudada, objetivos do estudo, instrumentos utilizados, resultados e as conclusões com base nas consequências para o trabalhador e a instituição.

\section{Resultados}

No período estudado, foram encontrados 59 estudos, sendo selecionados sete artigos que se enquadram nos critérios preestabelecidos, sendo quatro produções internacionais e três publicações nacionais, que se encaixaram nos critérios para inclusão deste estudo (Figura 1), os quais se encontram sumarizados com base em suas características na Tabela 1 .

Os estudos selecionados envolveram graduandos, residentes, auxiliares de enfermagem e profissio- nais de nível superior. O tamanho amostral variou de 68 a 4.307 sujeitos e, no total, foram investigadas 6.172 pessoas nos sete estudos analisados. Entre os estudos encontrados, a expressiva maioria dos profissionais de enfermagem era do sexo feminino.

Ao analisar as metodologias utilizadas nas pesquisas, observou-se que os estudos utilizaram, mas não exclusivamente, abordagem quantitativa dos dados.

Os instrumentos utilizados para avaliação da depressão entre os profissionais de enfermagem variaram, sendo o Inventário de Depressão de Beck (IDB) o mais utilizado (em quatro estudos). Foram utilizados, também, um questionário denominado Antonovsky SOC e um questionário de autorrelato anônimo, além de análises de prontuários médicos utilizada por um dos estudos.

Entre as variáveis de desfecho, a depressão foi investigada, acompanhada de outras variáveis como qualidade de vida (QV), transtornos mentais e comportamentais, síndrome de Burnout, ansiedade e estresse.

Figura 1 - fluxograma dos artigos selecionados

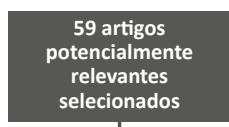

11 na base Lilacs

3 na base SciELO

45 na base PubMed 
Tabela 1 - características dos estudos selecionados, publicados entre 2000 a 2010, abordando a depressão no trabalho da enfermagem.

Autor e ano

População

Objetivos

Instrumentos

Furegato et al.

2010

Franco et al. 2005

Murofose e Marziale 2005

Ruggiero 2003

Santos et al. 2003

Tselebis et al.

2001

Greenglass e Burke 2000
4.307 enfermeiros de 23 unidades assistenciais da FH-MG

114 graduandos de enfermagem $(82,5 \%$ mulheres)

68 residentes de enfermagem

142 enfermeiras

99 graduandos de enfermagem $(97 \%$ mulheres)

79 enfermeiros

62 mulheres e 17 homens

1.363 enfermeiros de hospitais do Canadá
Verificar a prevalência de depressão, autoavaliação da saúde e fatores associados entre estudantes de enfermagem.

Avaliar a QV e a prevalência de sintomas depressivos nos residentes de enfermagem da EPM.

Questionário de QV SF-36 IDB

Descrever a frequência de transtornos mentais e de comportamento em trabalhadores de enfermagem.

Prontuários médicos

Analisar transtornos depressivos entre enfermeiras de UTI.

IDB

Identificar e mensurar os sintomas de depressão entre os discentes de enfermagem.

IDB

IDB

Avaliar a relação entre Burnout, depressão e SOC entre enfermeiros.
Questionário Antonovsky 
Depressão presente em 15,4\% no bacharelado e $28,6 \%$ na licenciatura

Os aspectos relacionados à QV comprometidos no componente mental do SF-36: aspectos emocionais, vitalidade e saúde mental. Houve prevalência de depressão em $27,9 \%$ dos residentes de enfermagem.

Foram identificadas 692 consultas com diagnósticos relacionados a transtornos mentais e comportamentais, desses, 40,8\% foram diagnósticos de depressão entre os profissionais de enfermagem.

23\% das enfermeiras preencheram os critérios para depressão clínica. Não houve diferenças entre os turnos diurno ou noturno.

$41,41 \%$ apresentaram grau de depressão variando de leve até grave. Os sintomas mais frequentes foram autoacusação, irritabilidade e fadiga.

Houve correlação entre SOC com Burnout e depressão, e quanto maior o grau de depressão menor o nível de Burnout e SOC.

A quantidade de trabalho foi um estressor consistente e significativo. A maior carga de trabalho da enfermeira associada à maior exaustão emocional levou a quadros de depressão e ansiedade.
Decresce a depressão conforme cresce o nível econômico na licenciatura. A autoavaliação mental teve as razões de prevalência significativas para depressão.

A população de residentes de enfermagem precisa ser mais estudada, ouvida e valorizada. A melhoria das condições de trabalho refletirá na assistência de enfermagem ao paciente e na QV de seus sujeitos.

A presença de episódios depressivos que afetam principalmente assistentes de enfermagem merece atenção especial no local de trabalho em que esses trabalhadores desempenham características especiais que as tornam mais vulneráveis.

Apesar da prevalência de depressão ter sido baixa, sugerese a necessidade de estratégias para promover o sono e melhora do humor de enfermeiros que trabalham em UTI.

A investigação aponta para a necessidade de programas que acompanhem o aluno durante sua formação acadêmica.

O grau de SOC torna os enfermeiros vulneráveis ou resistentes à depressão e Burnout.

Os resultados do estudo sugerem maior sofrimento psicológico e menos segurança no trabalho. 
Os estudos selecionados por esta revisão identificaram algum grau de depressão ou comprometimento na saúde mental dos profissionais de enfermagem, desde os graduandos, passando pelos residentes, até os profissionais que já estão atuando na área, principalmente, aqueles que atuam em nível hospitalar, seja no âmbito de terapia intensiva ou enfermarias, porém não foi possível observar média de prevalência do distúrbio estudado, em virtude de alguns estudos não apresentarem valores exatos.

Foram identificados vários fatores associados à ocorrência de depressão nas pesquisas analisadas, os quais podem ser subdivididos em fatores desencadeantes internos e externos ao ambiente de trabalho, que trazem consequências para o trabalhador e a instituição hospitalar.

\section{Discussão}

O presente estudo identificou artigos científicos publicados em revistas nacionais e internacionais indexadas, que identificaram algum grau de depressão relacionada ao trabalho de enfermagem no período pesquisado.

Os trabalhadores de enfermagem, em sua atividade laboral, encontram-se expostos a psicopatologias, como a depressão, em decorrência da relação entre o trabalho hospitalar e a saúde e, mais especificamente, o trabalho hospitalar e a saúde mental do profissional (MANETTI; MARZIALE, 2007). Essa relação expõe os trabalhadores fisicamente, aos riscos químicos, às radiações, às contaminações biológicas, ao excesso de calor, ao sistema de plantões, à excessiva carga horária de trabalho e à organização hierárquica do trabalho de enfermagem; e psiquicamente, decorrente da convivência diária com o sofrimento, a dor, a doença e a morte, tendo que assimilar tais circunstancias paralelamente aos seus problemas emocionais (MANETTI; MARZIALE, 2007).

Entre os sujeitos avaliados pelos estudos que compuseram esta revisão, pode-se observar que variaram entre estudantes de graduação, residentes, auxiliares de enfermagem e profissionais com ensino superior completo em enfermagem, e a maioria era do sexo feminino, apresentando algum grau de depressão. Outros estudos encontram prevalência média de depressão, em torno de $5 \%$ a 10\%, na população em geral (FUREGATO; SANTOS; SILVA, 2010) e de 10 a 25\% entre mulheres (KESSLER et al., 1993). Historicamente, a enfermagem tem sido uma profissão composta por mulheres na maioria dos países
(BOOTH, 2002), o que pode subsidiar os índices encontrados nos estudos enquadrados nesta revisão.

Ao analisar os instrumentos para avaliação do nível de depressão, utilizados pelos estudos selecionados por esta revisão, observou-se que o IDB foi o mais utilizado. Esse instrumento, amplamente reconhecido e utilizado em diversos países do mundo, avalia a intensidade de depressão e pode ser aplicado em pacientes psiquiátricos ou na população não clínica (GORENSTEIN; ANDRADE, 1996), porém não avalia todas as dimensões que envolvem a saúde mental de uma pessoa, podendo esta ser uma limitação dos estudos que o utilizaram.

Por meio da análise de conteúdo dos resultados encontrados, observou-se que a saúde mental dos trabalhadores de enfermagem pode ser influenciada por fatores internos ao ambiente e processo de trabalho e por fatores externos. Entre os fatores internos ao ambiente e processo de trabalho da enfermagem, a literatura científica aponta que alguns setores de trabalho hospitalar, como a Urgência e Emergência (UE), a Unidade de Terapia Intensiva (UTI) e o Centro Cirúrgico (CC), são mais desgastantes, implicando diminuição da QV do trabalhador e aumentando sua predisposição a transtornos mentais e processos depressivos (FRANCO; BARROS; MARTINS, 2005).

Ao analisar a relação da saúde mental com o trabalho, a falta de condições, a organização e a dificuldade de remanejamento, a possibilidade de participação na gestão e a concepção da tarefa correlacionam-se e interferem na saúde psíquica dos trabalhadores (CAMAROTTI; TEIXEIRA, 1996).

Os problemas de relacionamento com supervisores e médicos vivenciados pela equipe de enfermagem acarretam em maior sofrimento relacionado ao trabalho, menor satisfação no trabalho, menor intenção de permanecer no emprego, e humor deprimido. Em longo prazo, esses problemas podem estabelecer fator de risco para o desenvolvimento de depressão e problemas físicos (SCHAEFER; MOOS, 1996).

O sofrimento psíquico do trabalhador está associado ao desgaste no trabalho, ao apoio social insuficiente, ao sentimento de insegurança no trabalho e à instituição de atuação do profissional (BOURBONNAIS et al.,1998). A depressão, avaliada em trabalhadores de enfermagem no Caribe, apresentou correlação positiva com a sobrecarga de trabalho e o conflito de interesses e correlação negativa com apoio social, entendido como o 
suporte oferecido pelos superiores, colegas de trabalho e familiares (BABA; GALAPERIN; LITUCHY, 1999).

Em relação aos fatores externos ao trabalho de enfermagem, a associação direta entre a renda familiar mensal e o aumento do nível de depressão foi observada em estudo realizado com graduandos de enfermagem, entre os quais se observou ainda que os eventos novos continham conotação de negatividade, os quais eram enfrentados com muito pessimismo, o que explicaria a alta presença de sintomatologia depressiva (41\%) entre os estudantes (SANTOS et al., 2003).

Ao avaliar a QV e a prevalência de sintomas depressivos em residentes de enfermagem, foi observado que os aspectos emocionais, a saúde mental e a vitalidade eram as dimensões mais comprometidas do estado geral de saúde dessas pessoas, e que $27,9 \%$ dos residentes apresentavam aspectos de prevalência de depressão (FRANCO; BARROS; MARTINS, 2005). Esses achados demonstram a relação direta entre os transtornos de SOM, como a depressão, e piores níveis de QV, que podem afetar diretamente as atividades profissionais e sociais das pessoas acometidos.

O presente estudo apresentou algumas limitações importantes que merecem ser destacadas: a inclusão de trabalhos que avaliaram os profissionais dessa classe em diferentes momentos de sua formação desde a graduação, passando pela residência até os profissionais já efetivados; a heterogeneidade nos instrumentos de avaliação da depressão, o que pode subestimar ou superestimar os níveis reais desse acometimento, tornando limitadas possíveis comparações; a exclusão de alguns estudos por dificuldade na obtenção destes, ou seja, a ausência de disponibilidade.

Com base nas pesquisas analisadas, identificou-se que a depressão entre profissionais de enfermagem pode estar correlacionada positivamente com exaustão emocional e negativamente com senso de coerência. Esses achados confirmaram a hipótese de que o grau do senso de coerência pode acarretar em vulnerabilidade ou resistência da enfermagem à depressão e ao Burnout (TSELEBIS; MOULOU; ILIAS, 2001).

\subsection{Estratégias de prevenção}

As intervenções para a redução do sofrimento psíquico no trabalho estão associadas a melhor e mais clara divisão do trabalho, entre os trabalhadores de enfermagem e os demais profissionais da saúde; a reposição dos trabalhadores faltantes, para manter a eficiência de cada equipe de trabalho; o apoio do supervisor e dos colegas quando a solução de problemas na clínica; o reconhecimento por parte dos superiores; a participação no processo de tomada de decisão; a oportunidade para desenvolver suas habilidades; e oportunidades para falarem sobre as tensões no trabalho (MANETTI; MARZIALE, 2007).

As pesquisas revelaram a necessidade da implantação de programas de atenção à saúde dos trabalhadores que envolvam grupos de discussão, grupos de vivências, psicoterapia e administração participativa, com utilização de estratégias apropriadas, visando à minimização do estresse e do Burnout, ao gerenciamento da depressão relacionada ao trabalho, à redução do absenteísmo e da rotatividade entre os trabalhadores de enfermagem, o que reforça a necessidade de medidas de acompanhamento, por meio de programas preventivos e de tratamento (SANTOS et al., 2003; TRINDADE et al., 2010).

\section{Considerações Finais}

Este estudo possibilitou identificar, em forma de síntese das informações disponíveis, a depressão como problema de saúde que tem acometido, com frequência, os trabalhadores de enfermagem. Apesar do pequeno número de artigos divulgados nas principais bases de dados nacionais e internacionais, os resultados obtidos nas pesquisas realizadas contribuem, sobremaneira, com o avanço do conhecimento cientifico e como incentivo para a realização de novas pesquisas.

\section{Referências}

BABA, V.; GALAPERIN, B.L.; LITUCHY, T.R. Occupational mental health: a study of work-related depression among nurses in the Caribbean. International Journal of Nursing Study, Philadelphia, v. 36, n. 2, p. 163-169, Apr. 1999. doi: 10.1016/S0020-7489(99)00002-4

BOOTH, R. Z. The nursing shortage: a worldwide problem. Revista Latino Americana de Enfermagem, Ribeirão Preto, v. 10, n. 3, p. 392-400, maio/jun. 2002. doi: 10.1590/S0104-11692002000300013

BORGES, A. M. B.; CARLOTTO, M. S. Síndrome de Burnout e fatores de estresse em estudantes de um curso técnico de enfermagem. Aletheia, Canoas, n. 19, p. 45-56, jun. 2004. 
BOURBONNAIS, R. et al. Job strain, psychological distress, and burnout in nurses. American Journal of Industrial Medicine, New York, v. 34, n. 1, p. 20-28, jul. 1998. doi: $\quad$ 10.1002/(SICI)1097-0274(199807)34:1<20::AID-AJIM4>3.0.CO;2-U

CAMAROTTI, H.; TEIXEIRA, H.A. Saúde mental e trabalho: estudo da Regional Norte de Saúde do DF. Revista Saúde do Distrito Federal, Brasília, v. 7, n. 1, p. 29-40, jan./mar. 1996.

ELIAS, M. A.; NAVARRO, V. L. A relação entre o trabalho, a saúde e as condições de vida: negatividade e positividade no trabalho das profissionais de enfermagem de um hospital escola. Revista Latino Americana de Enfermagem, Ribeirão Preto, v. 14, n. 4, p. 517-525, jul./ago. 2006. doi: 10.1590/S0104-11692006000400008

FRANCO, G. P.; BARROS, A. L. B. L.; MARTINS, L. A. N. Qualidade de vida e sintomas depressivos em residentes de enfermagem. Revista Latino Americana de Enfermagem, Ribeirão Preto, v. 13, n. 2, p. 139-144, mar./abr. 2005. doi: 10.1590/S0104-11692005000200002

FUREGATO, A. R. F; SANTOS, J. L. F.; SILVA, E. C. Depressão entre estudantes de dois cursos de enfermagem: autoavaliação da saúde e fatores associados. Revista Brasileira de Enfermagem, Brasília, v. 63, n. 4, p. 509-516, jul./ago. 2010. doi: 10.1590/S0034-71672010000400002

GORENSTEIN, C.; ANDRADE, L. Validation of portuguese version of the Beck depression inventory and the state-trait anxiety inventory in brazilian subjects. Brazilian Journal of Medical Biological Reserch, São Paulo, v. 29, p. 453-457, mar. 1996. doi: 10.1590/S0100879X2001000300011

GREENGLASS, E. R.; BURKE, R. J. Hospital downsizing, individual resources, and occupational stressors in nurses. Anxiety Stress Coping: an International Journal, Toronto, v. 13, n. 4, p. 371-390, jul./ago. 2000. doi: $10.1080 / 10615800008248342$

JODAS, D. A.; HADDAD, M. C. L. Síndrome de Burnout em trabalhadores de enfermagem de um pronto socorro de hospital universitário. Acta Paulista de Enfermagem, São Paulo, v. 22, n. 2, p. 192-197, mar./abr. 2009. doi: 10.1590/S0103-21002009000200012
KESSLER, R. C. et al. Sex and depression in the National Comorbidity Survey I. Lifetime prevalence, chronocity and recurrence. Journal of Affective Disorders, Amsterdam, v. 29, n. 2-3, p. 85-96, Oct./Nov. 1993. doi: 10.1016/0165-0327(93)90026-G

MANETTI, M. L.; MARZIALE, M. H. P. Fatores associados à depressão relacionada ao trabalho de enfermagem. Estudos de Psicologia (Natal), Natal, v. 12, n. 1, p. 79-85, jan./abr. 2007. doi: 10.1590/S1413-294X2007000100010

MUROFUSE, N. T.; MARZIALE, M. H. P. Trastornos mentales y de comportamiento en trabajadores de enfermería de 23 instituciones de salud en Brasil. Revista de Enfermería del IMSS, Cidade do México, v. 13, n. 3, p. 133-140, set./dez. 2005.

PAFARO, R. C.; MARTINO, M. M. F. Estudo do estresse do enfermeiro com dupla jornada de trabalho em um hospital de oncologia pediátrica de Campinas. Revista da Escola de Enfermagem da USP, São Paulo, v. 38 , n. 2, p. 152-160, jun. 2004. doi: 10.1590/S008062342004000200005

RUGGIERO, J. S. Correlates of fatigue in critical care nurses. Reserch in Nursing \& Health, New York, v. 26, n. 6, p. 434-444, Dec. 2003. doi: 10.1002/nur.10106

SANTOS, T. M. et al. Aplicação de um instrumento de avaliação do grau de depressão em universitário do interior paulista durante a graduação de enfermagem. Acta Scientiarum Health Sciences, Maringá, v. 25, n. 2, p. 171-176, jul./dez. 2003. doi: 10.4025/actascihealthsci.v25i2.2228

SCHAEFER, J. A.; MOOS, R. H. Effects of work stressors and work climate on long-term care staff's job morale and functioning. Reserch in Nursing \& Health, New York, v. 19, n. 1, p. 63-73, Feb. 1996. doi: 10.1002/(SICI)1098-240X(199602)19:1<63::AID-NUR7>3.0.CO;2-J

TRINDADE, L. L. et al. Estresse e síndrome de Burnout entre trabalhadores da equipe de Saúde da Família. Acta Paulista de Enfermagem, São Paulo, v. 23, n. 5, p. 684-689, set./out. 2010. doi: 10.1590/S0103-21002010000500016

TSELEBIS, A.; MOULOU, A.; ILIAS, I. Burnout versus depression and sense of coherence: study of greek nursing staff. Nursing \& Health Sciences, Malden, v. 3, n. 2, p. 69-71, June 2001. doi: 10.1046/j.1442-2018.2001.00074.x 